\title{
An Improved HDR Technique by Classification Method
}

\author{
Deepali Agarwal \\ M.tech Dept. of CSE \\ VITM Gwalior \\ Gwalior, India
}

\author{
Shilky Shrivastava \\ Asst prof. Dept. of CSE \\ VITM Gwalior \\ Gwalior, India
}

\author{
Anand Singh Bisen \\ HOD. Dept. of CSE \\ VITM Gwalior \\ Gwalior, India
}

\begin{abstract}
Now a day, HDR image is mostly used in applications of wide range such as next generation broadcast, digital cinema and digital photography, because of its high quality and its good expression ability. In our work we have improved an HDR (High Dimensional image) and changed it into multiple improved Low Dynamic Range (LDR) images. We further used multiple copies of the same image and classified differently as per new Low Dynamic Range. Hence we obtain many new exponentially improved images. These images can be further improved by further LDR technique. Finally, we combine all these images and obtain a final image. This final image will be better as compared to the original image. Hence we have an image which in itself will be improved version of the given image.
\end{abstract}

\section{Keywords}

High dynamic range imaging, HDR, Histogram stretching, Denoising.

\section{INTRODUCTION}

Acquisition of realistic photographs becomes easier for onexperts since high-quality imaging devices are popular in consumer electronics market. Three fundamental factors for realistic acquisition include; i) high spatial resolution, ii) high dynamic range (HDR), and iii) accurate color reproduction. The HDR imaging technique has recently emerged in recent years and played an important role in bringing a new revolution to digital imaging [1]. While human vision can recognize a wide range of brightness levels over 100,000, digital imaging devices have a limited dynamic range because of the finite number of bits of pixel intensity values. To overcome the limited dynamic range, the most popular HDR imaging technique acquires multiple, differently exposed input images and appropriately fuses them to obtain the extended dynamic range and color gamut [2] [3]. Whereas the multiple image-based techniques can successfully provide HDR images in the static scene, it produces ghost artifacts if an object moves during the exposure time. Moreover, it is even more difficult to acquire motion-free multiple frames using a compact mobile camera with limited computational power. For this reason, a single frame HDR algorithm is needed for consumer mobile imaging devices. On the other hand, Land has proposed the Retinex image processing method, which recovers color by estimating light source and reflectivity [4]. However, color distortion and noise are amplified during the recovery process.

Typical cameras represent a pixel using only 256 values for each of the red, green, and blue channel. On the contradictory, the range of radiance of actual scene has a far wider range than 256 values [5]. Hence, a photograph captured by a conventional camera cannot capture the whole dynamic range of scene radiance. So, the cameras usually compress the scene radiance value using a proper function which is often called the camera response function (CRF). This process, however, can cause unpleasant under- or overexposed regions. Many approaches have been proposed to recover a high dynamic range image (HDRI) by estimating the CRF using multiple low dynamic range images (LDRIs) which are taken under different exposure settings for the scene [6].

\subsection{Applications}

Our method of the high dynamic range radiance maps recovering and also deriving imaging response functions in computer graphics contains many possible applications:

\subsubsection{Image-based rendering and modeling}

Image-based rendering systems and modeling to date (e.g. [713]) create the assumption that each of the images are taken with the similar settings of exposure and also response about film functions. However, closely any extensive atmosphere will have certain areas that are much brighter than others, creating it incredible to adequately photograph the scene applying a set of single exposure. In inside scenes with windows, this situation often rises within the field of vision of a single photograph, since the areas visible by the areas inside the building are less bright and also windows can be far brighter. By determining the response functions of the imaging device, the method presented here permits one to correctly fuse pixel document from the photographs that taken at the settings of dissimilar exposure. As an outcome, one can suitably photograph outdoor areas with small exposures, and indoor areas with larger exposures, without making inconsistencies in the data set. Also, knowing the response functions can be helpful immerging photographs taken with different imaging systems, like video cameras, digital cameras, and film cameras with processes of digitization and numerous stocks of film.

The area of rendering and image-based modeling is working toward improving the models of advanced reflection of the planes in the scene (e.g. [14]). These approaches, which contain identifying surface radiance in numerous directions under numerous lighting conditions, need complete values of radiance rather than finding the values of mapped pixel nonlinearly in conventional images. Just as important, the retrieval of high dynamic range images will permit these approaches to find correct radiance values from surface secularities and from incident light sources. Such higher radiance values typically become clamped in conventional images. 


\subsubsection{Image processing}

Most image processing operations, such as edge detection, blurring, image correspondence, and color correction, assume the values of the pixel to be proportional to the radiance scene. Because of the nonlinear image reply, particularly at the point of saturation, these processes can create improper outcomes for images conventional. In the computer graphics, one general image processing, the procedure is the application motion of synthetic blur to the images.

\subsubsection{Image compositing}

In computer graphics involve the compositing, image data from images obtained by different methods. For example, a matter of background might be shot with the camera, live performance might be shot with the CG elements or dissimilar film stock and scanning procedure would be created by rendering procedures. When there are significant alterations in the replay curves of these imaging procedures, the composite image can be visually implausible. The technique offered in this paper provides a convenient and robust technique of defining the total response curve of any imaging process, allowing images from different processes to be used consistently as radiance maps. Similarly, the recovered response curves can be reversed to the render composite radiance map as if it had been photographed with one of the processes of original imaging, or a disparate imaging process entirely.

\subsubsection{A research tool}

One aim of the computer graphics is to simulate the image creation, developed in a way that creates the outcomes that are consistent with what occurs in the actual world. Rearranging radiance maps of the actual scenes should permit the quantitative evaluations of the rendering procedures to be made in addition of the qualitative examination they traditionally receive. In certain, the method should be useful for developing reflectance and illumination models, and comparing of global illumination solutions against ground truth data. On condition that high dynamic range scenes on the convention show Devices is the issue of the considerable preceding work, containing $[15,16]$. The work obtainable in this paper will permit such approaches to be tested on the actual radiance maps in the addition to computed synthetically radiance results.

\section{HDR IMAGE RENDERING ALGORITHM}

HDR image rendering procedures can be approximately categorized by the processing of the spatial methods into the two clusters: local and global operators [Reinhard et al. 2006]. The global operator applies the similar type transformation to each pixel in the image which is based on the content global image, even though for the local operators a particular mapping tactic is used for every pixel based upon its spatially localized content. There are weaknesses and strengths to both the local and global approaches tone-mapping. The global operators tend to be the computationally simpler and as an outcome can be simpler to the faster to execute and implement also. The local operators tend processing to be computationally additional exclusive, but can permit for the dramatic decrease in generally dynamic range. From the opinion of rendering aims or in tents, certain procedures purpose for the images produce that are visually appealing, applying the digital image processing and photographic methods to the enhance rendering pleasantness, while next procedures goal for the accuracy perceptual. These algorithms try to the mimic perceptual abilities in addition to the compressing the range of luminance, causing in an image which provokes the similar visual reply as a human may have when observing the similar actual scene. Other algorithms are aimed to the maximize general discernibility in images; an example would be to use of HDR medical images. Since the local algorithms are efficient of the large dynamic range of compression and also tend to mimic of the local adaptation, performance of the human visual method, more emphasis was put on an examination more of these kinds of the algorithms exactly. A brief overview of the algorithms is given below:

\subsection{Sigmoidal Transformation}

Braun [Braun and Fairchild 1999] obtainable a lightness rescaling method in image for gamut mapping of the 8-bit images using the enhancement function of sigmoid contrast. The system of sigmoid functions was resulting from a normal function of the discrete cumulative, given in Eq. 1, where $\mathrm{x} 0$ and are the variance and mean of a normal distribution. While this technique was valuable for the gamut mapping, applying the lightness channel in a space, color appearance, such as CIELAB, it was not conscious for the extreme dynamic range decrease. The actual implementation was changed so that instead of "lightness", so the luminance logarithm, normalized from 0 to 100, was used to compress of the HDR images.

$$
S_{i}=\sum_{n=0}^{n=i} \frac{1}{\sqrt{2 \pi \sigma}} e^{\frac{\left(x_{n}-x_{0}\right)^{2}}{2 \sigma^{2}}}
$$

\subsection{Histogram Adjustment}

Ward [Ward Larson et al. 1997] presented the reproduce global operator to the perceptually correct tones in the HDR scenes by the determining luminance adaptation, which is local in levels and also altering the histogram luminance. The human visual models of the color sensitivity, glare and spatial acuity special effects are incorporated into this model to the reproduce imperfections in the mimic the subjective and human vision observing knowledge.

\subsection{Retinex}

"A Retinex is totally mechanisms from the retina to the cortex necessary to form images in the positions of lightness" [McCann 2004]. The use of real image dynamic range compression was originally defined in a patent by the [Frankle and McCann 1983]. We check about the McCann99 version of the Retinex with the public code of Matlab prepared accessible by [Funtet al.2000]. An automatic technique [Funt et al. 2002] was used for setting the no. of the iterations, a parameter which is free from compression of the dynamic range resulting image in Retinex and controls contrast.

\section{4 iCAM}

iCAM [Johnson and Fairchild 2003] is an image entrance model that has been stretched to the high dynamic range images render for the purpose of the presentation. iCAM attempts to the predict complex human visual replies by the joining classical color presence abilities with the properties of spatial vision.

\subsection{Photographic Reproduction}

Reinhard et al. [Reinhard et al. 2002] obtainable a tone duplicate method for the HDR rendering by the simulating 
burning-and-dodging in classical photography. This technique mechanically applies dissimilar luminance mapping scales to the shadow regions and relative highlight, where the local contrast is projected using the center-surround function with dissimilar spatial extent.

\subsection{Bilateral Filtering Technique}

Dorsey and Durand [Dorsey and Durand 2002] suggested a rendering method to decrease the generally contrast, while local details in the image are preserved. Decomposes the image into two layers, edge-preserving spatial processing operator, termed a bilateral filter: first of the base layer, largescale variations encoding, and layer detail is the second. The contrast of the layer base is compressed and they are also joint again with the detail layer to create the final image. This method was improved slightly use to the CIE XYZ colorimetric to calculate the luminance channel. Whole brightness and base contrast, parameters of two usercontrolled, were set to 1.4 and 1.8 respectively. An extract to the 1st and 99th percentile of the image rendering document was achieved to eliminate some extremely bright and dark pixels prior to the presentation.

\subsection{Modified iCAM}

The Bigg's modified iCAM [Biggs 2004] incorporates Reinhard and Devlin's tone mapping operator into the $\mathrm{ICAM}$ framework, which is recognized on the physiology of the photoreceptors in the human eye. The operator outcome is certain grouping of a globally adapted value which is based on locally adapted value around all image pixel and image averages. This technique goals to the reservation the chromatic adaptation and color fidelity of iCAM while protective the tone reproduction capabilities of Devlin's and Reinhard operator.

\subsection{Local Eye Adaptation}

The method local-eye adaptation [Ledda et al. 2004] compresses the dynamic range of luminance channel of the simulating the photoreceptor responses in the retina. The electro physiological equation, well-known as equation of the Naka-Rushton [Naka and Rushton1966], was adapted to the local operator that predicts S-shaped reply function of the cones and rods at intensities of localized adaptation. The bilateral filter was used to average local luminance to avoid halo common artifacts to the other local operators.

\subsection{Retinex-Based Adaptive Filter}

Surround Retinex model based on adaptive filter for the HDR rendering. The luminance channel is then preserved by surround-based Retinex approaches, which is defined by main component of the examination about principal component. Examination about Principal component offers orthogonally between the channels and decreases the chromatic modifications produced by the alteration of the luminance. The weights of the surrounding pixels are computed with an adaptive filter technique by the adapting shape of the filter to the high contrast edges in the images to prevents halo artifacts, one of the significant difficulty of the previous surround-based Retinex approaches. Before going on, it is significant to the stress and goal of these evaluations is to improve improved understanding on how current HDR rendering algorithms achieve in terms of image rendering accuracy and preference, not to the select the finest general procedure. The results of the trial should be helpful for the surveys of the HDR rendering techniques, especially in the tone mapping and spatial processing. The aim is to guide the expansion of more robust HDR rendering procedures. The relationship between the image overall visual accuracy or pleasantness and preference was reconnoitered in this study. The significance of the understanding this relationship lies in the definition whether separate procedures are necessary for different rendering intents, such as accurate or pleasing rendering. In addition, it would be useful to discover a algorithm which is common or default that could serve as a baseline TMO for the future algorithm testing trials, Such as the work of CIE Technical Committee 8-08 on testing spatial color appearance models [Johnson 2005].

\section{RELATED WORK}

Capturing the entire dynamic range of a scene using a single image is a challenging problem in computational photography. Analog cameras can capture such scenes with a very high dynamic range using a single snapshot. The common digital cameras are not capable of doing so due to the limited dynamic range of the sensor. However, one can capture multiple, differently exposed images of the scene and composite them in the irradiance domain and capture the entire dynamic range [17]. There are digital cameras which can capture the entire dynamic range of the scene using a single snapshot [18]. These cameras are very expensive in the market. In this section, we would address some of the works done earlier in HDR imaging along with an overview of the segmentation techniques. Mann and Picard introduced a method to recover the camera response function (CRF) and estimate the HDR image from multi exposure images [19]. They used the derivative of the CRF, called certainty function, to weigh the multi-exposure irradiances. Debevec and Malik developed a practical algorithm for recovering the HDR image and used a simple hat function as the weighting function [20]. Mitsunaga and Nayar estimated the CRF by parameterize and then generated the HDR image [21]. CRF can also be recovered from a single image provided the image has edges of highly varying magnitudes [22]. An overview of all these different methods for the generation of HDR image from multi-exposure images of a static scene can be found in [22]. Assuming CRF is known, Granados et al recently developed a method to generate an HDR image in the presence of various types of noise [23].

The generated HDR image can then be encoded in Radiance RGBE (.hdr) or Open EXR (.exr) formats which employ floating point numbers to store the intensity values. These image formats require a large amount of memory and need to be compressed for optimal storage. The approaches discussed above for the generation of HDR image have to be complemented by an appropriate tone mapping operator for visualization in common displays and printers.

An example of tone mapping is the gradient domain HDR compression method by Fattal et al [23]. An overview of the different types of tone mapping operators (global and local) can be found in [22]. For a static scene, there are alternate methods based on digital compositing, which combine the multi-exposure images directly without the knowledge of CRF. These methods employ basic digital compositing principles with an appropriate weighting function.

The basics of digital compositing methodology can be found in([25], [26], [27], [28]). An interactive method for compositing image regions was proposed by Agarwala et al [31]. The method by Goshtas by uses entropy measures on blocks to combine multi-exposure images [32]. Exposure 
fusion combines multi-exposure images on a Laplacian pyramid using an appropriate weighting function [33].

A variational, iterative solution for combining multi-exposure images was introduced in [29]. Bilateral filter was used to define weighting function and composite multi-exposure images in [30].

While capturing multi-exposure images of a scene, we cannot guarantee that the scene would not change. There are chances of new objects being introduced in the scene between the exposures due to motion. Also, substances such as leaves and branches of a tree would move when there is the presence of heavy wind in the scene.

In other words, the scene would most probably be dynamic. When the methods mentioned above are employed for compositing multi-exposure images of a dynamic scene, the objects in motion in the scene would give rise to artifacts called ghosts. It is required that the changes in the scene are detected apriori before compositing is performed on the multiexposure images. We shall first look at the methods previously used for removing ghosting artifacts.

Jacobs et al. Proposed a method to identify the regions on the image grid, which change across multi-exposure images using weighted variance and entropy measures [34]. This method fills the motion regions by details from one of the observations and thereby reducing contrast in such regions. Gallo et al proposed a method to detect motion regions in multi-exposure images when CRF is known and eliminate them while compositing [35]. This approach preserves contrast in the motion regions as regions from multiple images are combined. An additional approach to eliminating ghosting artifacts while creating a mosaic of different image exposures was proposed in [36]. In this work, we address this problem from a bottom-up segmentation perspective.

A recent work assumed no knowledge of CRF and reconstructed the dynamic scene as an LDR image from multi-exposure images [37]. This method employs the gradient directions for detecting scene change and may perform poorly in the presence of even a small amount of noise of any form. Further, this approach cannot handle scenes which have tiny objects such as leaves of a tree in motion. Another problem is that this approach requires a number of parameters to be adjusted empirically.

Segmenting foreground from background in a single image is a classic vision difficulty. The segmentation algorithm can either be automatic or be interactive. One popular approach to achieve interactive segmentation is the Grabcut by Rother et al [38]. Interactive segmentation depends on the user input such as a bounding box or scribbles to perform segmentation. Another class of interactive algorithms which extract the alpha matte along with the foreground mask is recognized as matting. An example is the natural image matting[16]. We focus more on the automatic segmentation approaches in this work as we intend to develop an automatic method to compensate for scene change across different exposures.

Automatic segmentation approaches can be generally classified into top-down and bottom-up methods. In the topdown method, one attempts to capture the entire object boundary directly by the learned features of the desired object class ([4], [40]). This approach tries to get the foreground separated from the background as a whole. While in the bottom-up approach, the entire image is split into homogeneous regions based on color, contours, and texture details. These homogeneous regions are then grouped to segment the foreground from the background [39]. The bottom-up segmentation methods have derived much interest among the computer vision community of late as they lead to better segmentation of the foreground objects.

The algorithm by Shi and Malik uses normalized cuts to split a given image into multiple regions which are homogeneous [39]. This approach was later extended to define different homogeneous regions of the image as super pixels [41]. Each super pixel is a collection of a set of pixels inside a closed contour signifying uniformity in terms of color and texture. Object recognition systems can then work on the level of super pixels instead of image pixels which can help in designing faster algorithms. For segmentation tasks, one can group the super pixels belonging to foreground object based on some criteria to segment out the foreground. Even neighborhood can be defined for super pixels to improve the segmentation. Another approach to extract homogeneous regions from an image is the quick shift algorithm by Vedaldi and Soatto [42].

A typical bottom-up approach for segmentation relies on the efficient grouping of the super pixels and recovering the foreground. Apart from basic segmentation, grouping of super pixels has a number of applications in computer vision. Consider the problem of estimating the depth map of a scene from a single image. Super pixels corresponding to objects at different depths of the scene can be grouped to recover the 3D information from a single image [43].

By fast approximate background modeling the High dynamic range image deghosting [44] address ghosting by execution modification recognition on the exposure-normalized images, then decreasing the involvement of moving objects to the last compsite on the basis of frame-by-frame. Detection of Modification is computationally valuable and it can be useful for images exhibiting varied ghosting at the facts [44]. Demonstrate technique for both for HDR and LDR (Low Dynamic Range) images. Further constraints based on a priori information about the altering exposures HDR images apply. The stability rise of our method by applying recent super pixel segmentation techniques to enhance the change detection. In this solution contains a new method for areas that see motion throughout the capture, e.g., foliage gusting in the wind.

Elimination for high dynamic range images and Ghost detection: Recent advances [45], generally and a standard used method to find an HDR image is the multiple exposures' method of fusion which contains of the joining multiple images of the similar part with variable exposure times. However, if the scene is not static through the sequential acquisition, moving objects apparent themselves as ghosting artifacts in the final HDR image. Spotting and eliminating ghosting artifacts is an important subject for HDR images generating automatically of dynamic scenes. The goal of [45] is to deliver an up-to-date review of the suggested approaches for generation of ghost-free HDR image.

HTRI: High time ranges imaging's [46] have established a novel method to the image fusion from a sequence of the photographs of similar scene taken at different timestamps. When compared with HDRI, exposure bracketing at a single timestamp is swapped by timestamp difference disregarding exposure times. Because of the parallel among these two 
methods, this method is known as High Time Range Imaging (HTRI), it purposes of capturing ephemeral events happening over a period of long time during which an order of the images is shot. For every pixel location, the peak salient colors are privileged in the sequence of the photographs. The selection of the saliency standard is based on a study of the existing admitted definitions of visual attention. In a next stage, a higher priority is the assigned to pixels with high temporal saliency, i.e., which seems very briefly in the sequence, jointly generating spatial and temporal variations of contrast between two successive frames. [46] Algorithm captures each salient objects in the final image, without presenting a significant amount of noise, and without being affected by the larger illumination modifications that may happen in the acquisition conditions from one frame to the next. Experiments estimate the impact of the method parameters, and confirm profits of HTRI compared to other fusion techniques.

\section{PROPOSED METHODOLOGY}

\subsection{Algorithm}

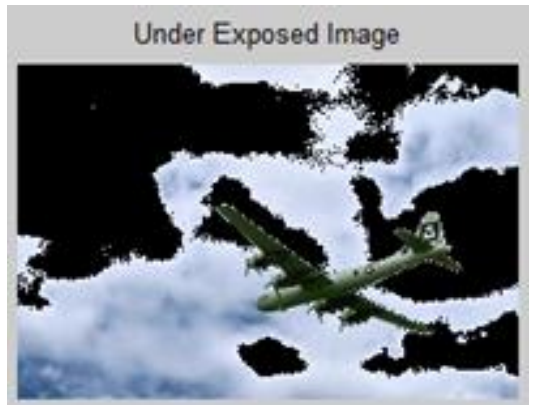

(a)

The various steps followed for proving our proposed concepts are:-

- Take ghosted Snap images captured by some sensing device.

- Now filter these images for further processing.

- (From here onwards repeat steps for each image)

- Apply HSV color transform to convert images into the hsv color scheme.

- Form different image patterns by altering the individual components in the previous result(i.e. Under exposed, over exposed, normal exposed).

- Apply Edge preserving denoising on each image obtained.

- Transform the individual images to rgb color model.

- Fuse three images into one to obtain the Sub HDR image.

- (After processing each image with above steps).

- $\quad$ Fuse the three obtained HDR images into one.

\section{RESULTS ANALYSIS}

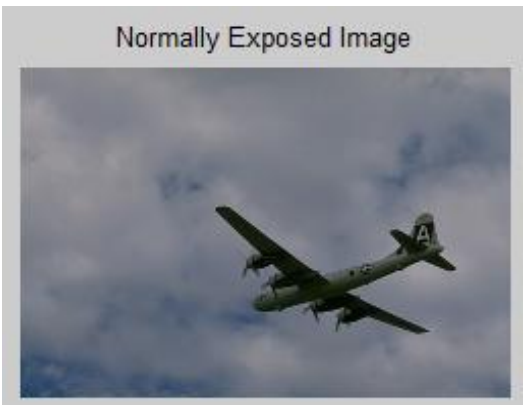

(b)

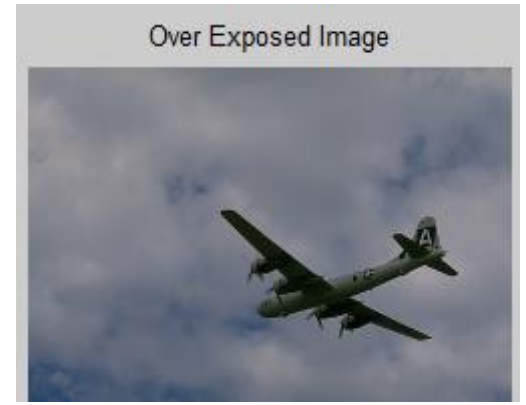

(c)

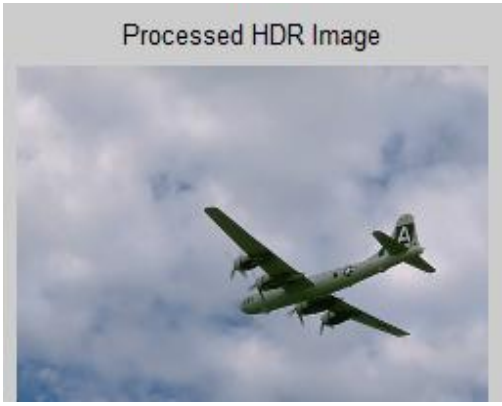

(d)

Fig 1: Normal HDR Processing 


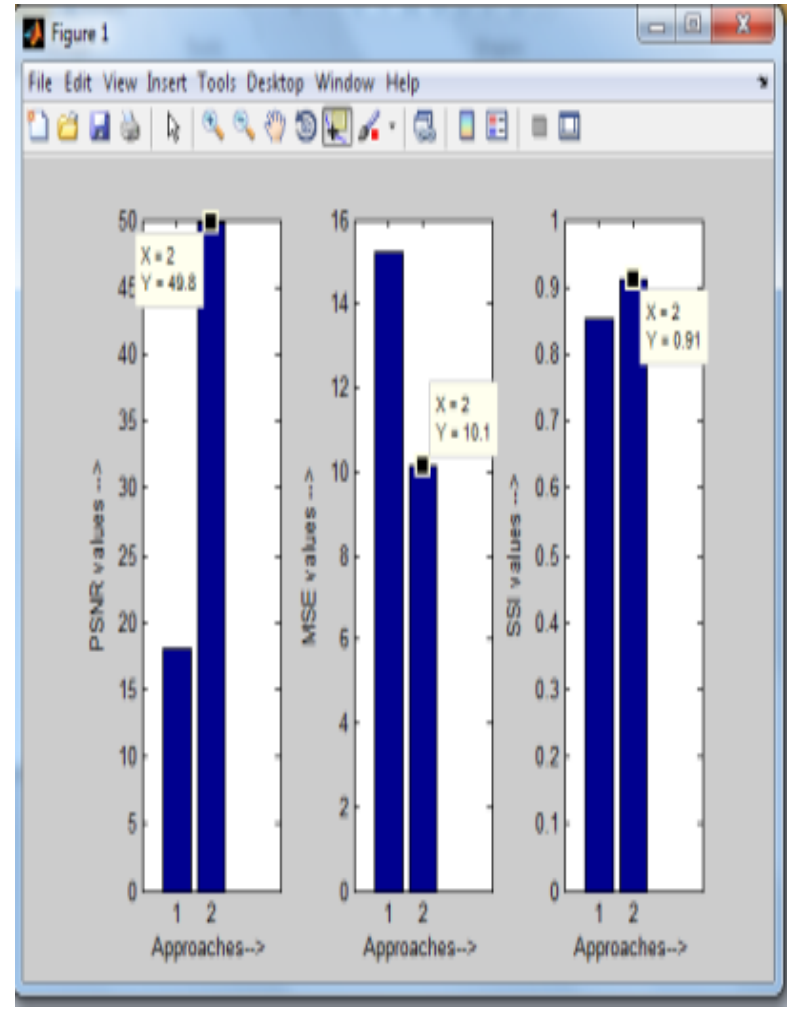

Fig 2: Graph shows the comparisons between base and proposed values of PSNR, MSE and SSIM.

Table1: In this table shows proposed model working on different images and calculate PSNR, MSE and SSIM

\begin{tabular}{|c|c|c|c|}
\hline $\begin{array}{c}\text { Image to be } \\
\text { processed }\end{array}$ & $\operatorname{PSNR}(\mathrm{dB})$ & MSE(unit) & $\begin{array}{l}\text { SSIM(par } \\
\text { ts) }\end{array}$ \\
\hline & 41.3 & 8.83 & 0.973 \\
\hline & 50.8 & 4.44 & 0.954 \\
\hline & 29.5 & 3.75 & 0.897 \\
\hline & 72.3 & 5.43 & 0.999 \\
\hline
\end{tabular}

\section{CONCLUSION}

Study in high dynamic range imaging has ongoing more than a decade ago, and it is a comparatively young field. Already, we have understood examples of the dramatic visual improvements quality that HDR image can attain over the classical low dynamic range image. Presently, this novel skill is still in its initial phase of the growth. In order to attain its full potential, for example, to implement HDR imaging technology in customer level digital camera to enable ordinary users to take high quality photographs and videos under any lighting conditions, there are still many technical hurdles to overcome, including, image sensing, coding and storage and display. This paper briefly present what have been attained in HDR imaging which also highlighted that much needs to be done in order to make this promising imaging knowledge the mainstay of the digital imaging. We have classified the images into low dynamic range images and then combined after application of the image processing techniques. This improves the quality of image. It can overcome camera's limitation of amount of color and luminance it can record is governed by the sensor's capability and the dynamic range of the camera's electronics. In future work we will take multiple input images and combine to produce HDR image. Also we merge Self organizing map with histogram separation method and we work on satellite images as well.

\section{REFERENCES}

[1] GORTLER, S. J.,GRZESZCZUK, R., SZELISKI, R., AND COHEN, M. F. The Lumigraph. In SIGGRAPH '96 (1996),pp. 43-54.

[2] LEVOY, M., AND HANRAHAN, P. Light field rendering. In SIGGRAPH '96 (1996), pp. 31-42.

[3] SZELISKI, R. Image mosaicing for tele-reality applications. In IEEE Computer Graphics and Applications (1996).

[4] WARD, G. J. Measuring and modeling anisotropic reflection. In SIGGRAPH '92 (July 1992), pp. 265-272.

[5] TUMBLIN, J., AND RUSHMEIER, H. Tone reproduction for realistic images. IEEE Computer Graphics and Applications13, 6 (1993), 42-48.

[6] SCHLICK, C. Quantization techniques for visualization of high dynamic range pictures. In Fifth Euro graphics Workshop on Rendering (Darmstadt, Germany) (June 1994), pp. 7-18.

[7] E. Reinhard, G. Ward, S. Pattanaik, and P. Debevec. High Dynamic Range Imaging: Acquisition, Display and Image-Based Lighting. Morgan Kaufmann Publishers, 2005.

[8] S. Nayar and T. Mitsunaga. High Dynamic Range Imaging: Spatially Varying Pixel Exposures. In IEEE Conference on Computer Vision and Pattern Recognition (CVPR), volume 1, pages 472-479, Jun 2000.

[9] S. Mann and R. W. Picard .On being un digital with digital cameras: extending dynamic range by combining exposed pictures. In Proc. of IS \& $\mathrm{T}$ 48th annual conference, pages 422-428, 1995.

[10] P. Debevec and J. Malik .Recovering high dynamic range radiance maps from photographs .In SIGGRAPH, 1997.

[11] T. Mitsunaga and S. K. Nayar. Radio metric self calibration. In CVPR, 1999. 
[12] G. Ward, E. Reinhard, S. Pattanaik, and P. Debevec, High dynamic range Imaging: acquisition, display, and image-based lighting, Morgan Kaufmann Publisher, 2005.

[13] Y. Bandoh, G. Qiu, M. Okuda, S. Daly, T. Aach, and O. $\mathrm{Au}$, "Recent advances in high dynamic range imaging technology," IEEE Conf. Int. Conf. Image Processing, pp. 3125-3128, 2010.

[14] P. Debevec and J. Malik, "Recovering high dynamic range radiance maps from photographs," Proc. ACM SIGGRAPH, pp. 369-378, August 1997.

[15] E. Land, "The retinex theory of color vision," Scientific American, vol. 237, pp.108-128, December 1977.

[16] Reinhard, E., Ward, G., Pattanaik, S., Debevec, P.: High dynamic range imaging: Acquisition, display and imagebased lighting. Morgan Kaufmann, San Francisco(2005)

[17] Jacobs, K., Loscos, C., Ward, G.: Automatic highdynamic range image generation for dynamic scenes. IEEE Computer Graphics and Applications 28, 84-93 (2008)

[18] LAVEAU, S., AND FAUGERAS, O. 3-D scene representationas a collection of images. In Proceedings of 12th International Conference on Pattern Recognition (1994), vol. 1, pp. 689-691

[19] MCMILLAN, L., AND BISHOP, G. Plenoptic Modeling: An image-based rendering system. In SIGGRAPH '95 (1995)

[20] CHEN, E. QuickTime VR - an image-based approach to virtual environment navigation. In SIGGRAPH '95 (1995).

[21] DEBEVEC, P. E., TAYLOR, C. J., AND MALIK, J. Modeling and rendering architecture from photographs: A hybrid geometry- and image-based approach. In SIGGRAPH '96(August 1996), pp. 11-20.

[22] S. Lin, J. Gu, S. Yamazaki, and H.-Y. Shum.Radio metric calibration from a single image. In IEEE CVPR, 2004.

[23] M. Granados, B. Ajdin, M. Wand, C. Theobalt, H. P. Seidel, and H. P. A. Lensch. Optimalhdr reconstruction with linear digital cameras. In IEEE CVPR, 2010

[24] R. Fattal, D. Lischinski, and M. Werman. Gradient domain high dynamic range compression. In SIGGRAPH, pages249-256, San Antonio, Texas, 2002.

[25] J. F. Blinn. Compositing, part 1: Theory. IEEE Computer Graphics \& Applications, 14(5):83-87, 1994.

[26] R. Brinkmann. The Art and Science of Digital Compositing. Morgan Kaufmann Publishers, 1999.

[27] T. Porter and T. Duff. Compositing digital images. InSIGGRAPH, pages 253-259, 1984.

[28] R. Szeliski. Image alignment and stitching: A tutorial. Foundations and Trends in Computer Graphics and Vision,2(1), 2008

[29] S. Raman and S. Chaudhuri.A matte-less, variational approach to automatic scene compositing. In ICCV, 2007.
[30] S. Raman and S. Chaudhuri. Bilateral filter based compositing for variable exposure photography. In EUROGRAPHICS Short Papers, 2009.

[31] A. Agarwala, M. Dontcheva, M. Agrawala, S. Drucker,A. Colburn, B. Curless, D. Salesin, and M. Cohen. Interactive digital photomontage. In SIGGRAPH, 2004

[32] A. Goshtasby. Fusion of multi-exposure images. Image and Vision Computing, 23:611-618, 2005.

[33] T. Mertens, J. Kautz, and F. V. Reeth. Exposure fusion: A simple and practical alternative to high dynamic range photography. Computer Graphics Forum, 28(1):161171,2009 .

[34] K. Jacobs, C. Loscos, and G. Ward. Automatic highdynamic range image generation for dynamic scenes. IEEE Computer Graphics and Applications, 28(2):84-93, 2008.

[35] O. Gallo, N. Gelfand, W. Chen, M. Tico, and K. Pulli. Artifact-free high dynamic range imaging. In ICCP, 2009.

[36] M. Uyttendaele, A. Eden, and R. Szeliski .Eliminating ghosting and exposure artifacts in image mosaics. In IEEECVPR, 2001

[37] W. Zhang and W.-K.Cham. Gradient-directed composition of multi-exposure images. In CVPR, 2010.

[38] C. Rother, V. Kolmogorov, and A. Blake. Grabcut: interactive foreground extraction using iterated graph cuts.ACM Trans. Graph., 23(3):309-314, 2004.

[39] J. Shi and J. Malik .Normalized cuts and image segmentation. IEEE Trans on PAMI, 22(8):888-905, 2000 .

[40] E. Borenstein and S. Ullman. Learning to segment. In ECCV,2004.

[41] X. Ren and J. Malik .Learning a classification model for segmentation. In IEEE ICCV, 2003.

[42] A. Vedaldi and S. Soatto.Quick shift and kernel methods for mode seeking. In ECCV, 2008.

[43] D. Hoiem, A. A. Efros, and M. Hebert. Automatic photopop-up. ACM Trans.Graph.,24(3):577-584, 2005

[44] Simon Silk, Jochen Lang "High dynamic range image deghosting by fast approximate background modelling" Computers \& Graphics 36 (2012) 1060-1071.

[45] Abhilash Srikantha ,De sire Sidibe" Ghost detection and removal for high dynamic range images: Recent advances" Signal Processing: Image Communication 27 (2012)650-662.

[46] J. Vis. Commun. Image "HTRI: High time range imaging" R. 24 (2013). 